\title{
THE LENSES STRUCTURE \& DYNAMICS SURVEY
}

\section{The internal structure and evolution of E/SO galaxies and the determination of $H_{0}$ from time-delay systems}

\author{
L.V.E. Koopmans ${ }^{1,2} \&$ T.Treu ${ }^{2}$ \\ ${ }^{1}$ Space Telescope Science Institute, 3700 San Martin Drive, Baltimore, MD 21218, USA \\ ${ }^{2}$ California Institute of Technology, mailcode 105-24, Pasadena, CA 91125, USA \\ koopmans@stsci.edu, tt@astro.caltech.edu
}

\begin{abstract}
The Lenses Structure \& Dynamics (LSD) Survey aims at studying the internal structure of luminous and dark matter - as well as their evolution - of field earlytype $(\mathrm{E} / \mathrm{SO})$ galaxies to $z \sim 1$. In particular, E/S0 lens galaxies are studied by combining gravitational lensing, photometric and kinematic data obtained with ground-based (VLA/Keck/VLT) and space-based telescopes (HST). Here, we report on preliminary results from the LSD Survey, in particular on (i) the constraints set on the luminous and dark-matter distributions in the inner several $R_{\text {eff }}$ of E/S0 galaxies, (ii) the evolution of their stellar component and (iii) the constraints set on the value of $\mathrm{H}_{0}$ from time-delay systems by combining lensing and kinematic data to break degeneracies in gravitational-lens models.
\end{abstract}

Keywords: gravitational lensing — distance scale — galaxies: kinematics and dynamics galaxies: fundamental parameters — galaxies: elliptical and lenticular, cD

\section{Introduction}

Even though massive E/S0 galaxies enclose most mass (luminous and dark) in the Universe on galactic scales, relatively little is observationally known about their their dark-matter halos or the evolution of their internal structure with time, and only recently studies have started to shed some light on the evolution of their stellar population with redshift.

The reasons for this are both observational and intrinsic to the mass modeling. First, since many of the studies of the mass distribution of E/S0 galaxies rely on stellar kinematics through spectroscopic observations, only with the advent of 8-10 m class telescopes (e.g. Keck and VLT) has it becomes possible to study E/S0 galaxies in any detail beyond the local Universe. Second, degeneracies in mass models that rely only on kinematic and photometric data often allow for multiple solutions and place limited or no constraints on the presence 
and distribution of dark-matter. These problems exacerbate with increasing redshift due to poorer observational constraints. Model degeneracies are often due to the unknown mass of the galaxy, allowing one to freely play with stellar anisotropy, the mass-density slope and its normalization. For example, approximately constant velocity dispersion profiles can be explained with an isothermal, kinematically isotropic, luminous plus dark-matter distribution, as well as a constant stellar $M / L$ model with a radially increasing tangential anisotropy. However, the latter model requires significantly less mass than the isothermal model within, say, several effective radii $\left(R_{\text {eff }}\right)$.

Hence, if the total mass of an E/S0 galaxy enclosed within some radius (around $\sim R_{\text {eff }}$ ) is known, one can break the degeneracy between the stellar $M / L$, the stellar anisotropy and the radial mass distribution. Strong gravitational lensing by E/S0 galaxies provides exactly the required information!

\section{The Lenses Structure \& Dynamics (LSD) Survey}

The 'clean' LSD Survey sample consists of 11 relatively isolated (e.g. no massive clusters nearby) E/S0 lens galaxies spread between redshifts $z=0.04$ and 1.01. The galaxies have a mass range of $\sim 1.5$ orders of magnitude. Multicolor photometric data is available in the HST archive (mostly from the CASTLeS collaboration) for each system (typically V, I and H bands). In 20012002, we obtained stellar kinematic data using the Echelle Spectrograph and Imager (ESI) on the Keck-II telescope with typically $0.7^{\prime \prime}$ seeing and under photometric conditions. Some systems have extended kinematic profiles (along major and sometimes minor axes), others only luminosity weighted dispersions, depending on their brightness and extent. Besides the clean sample, we also observed several other systems (also with the VLT), including several disk-galaxy lenses and lens systems with measured time-delays.

We continue with some of the high-lights of the LSD survey and related studies (e.g. the determination of $\mathrm{H}_{0}$ from lensing).

\section{The evolution of the stellar mass-to-light ratio}

Two LSD systems (MG2016+112 and 0047-285) have thus far been studied in detail (Koopmans \& Treu 2002, 2003; Treu \& Koopmans 2002a). Since we have available the (central) stellar velocity dispersion, effective radius and effective surface brightness (from the HST images; transformed to rest-frame B-band), each lens system can directly be compared with the local Fundamental Plane (FP). The offset from the FP is an indicator of the evolution of the effective surface brightness, due to fading of the stellar population with time (i.e. "passive evolution"). Both systems are consistent with passive evolution of field E/S0 galaxies, marginally faster than that of cluster E/S0 galaxies. We find that the stellar $M / L$ determined from the FP evolution and local measure- 
ments, being close to the "maximum-bulge solution", agree with those measured only from lensing and dynamics, suggesting that no structural evolution has occurred in the FP below $z \sim 1$.

\section{The luminous and dark-matter mass profile}

The combination of stellar kinematics and gravitational lensing can also be used to place stringent constraints on the luminous plus dark-matter mass profiles of E/S0 galaxies to $z \sim 1$. The reason is that lensing determines the mass inside the Einstein radius to a few percent accuracy. Varying the inner (total) mass slope - but satisfying the stringent mass constraint - leads to a considerable change in the observed line-of-sight stellar velocity dispersion profile, as well as the luminosity weighted stellar dispersion, of the E/S0 galaxy, only weakly dependent on details such as anisotropy, etc (see KT). Thence, a comparison with the observed stellar kinematics allows the determination of an 'effective' slope ( $\gamma^{\prime}$ for $\rho \propto r^{-\gamma^{\prime}}$; i.e. the average luminous dark-matter power-law slope) inside the Einstein radius (typically $1-5 R_{\text {eff }}$ ).

Thus far, we have found that $\gamma^{\prime}=2.0 \pm 0.1 \pm 0.1$ for MG2016+112 (Treu $\&$ Koopmans 2002a) and $\gamma^{\prime}=1.9_{-0.23}^{+0.05} \pm 0.1$ for 0047-285 (Koopmans \& Treu 2003; 68\% C.L. and syst. error). For B 1608+656-G1 and PG1115+080, both not part of the 'clean' LSD sample, we find $\gamma^{\prime}=2.03 \pm 0.14 \pm 0.03$ and $\gamma^{\prime}=2.35 \pm 0.1 \pm 0.05$, respectively (Koopmans et al. 2003; Treu \& Koopmans 2002b). E/S0 galaxies in the clean sample are both consistent with isothermal mass profiles (i.e. $\gamma^{\prime}=2$ ). From all four systems studied thus far, the average is $\left\langle\gamma^{\prime}\right\rangle=2.1$ with an $\mathrm{rms}$ of $0.2 ;$ E/SO galaxies appear on-average isothermal to within $\sim 10 \%$ inside several effective radii, eventhough some intrinsic scatter between systems is found, as expected.

We note that this is a preliminary results and the analysis of the full sample is required to confirm/strengthen this conclusion. Even so, E/S0 galaxies require a considerable diffuse dark-matter component inside the stellar spheroid in order to explain the observed stellar kinematics; constant stellar $M / L$ models are excluded with high confidence in all cases. The luminous plus dark-matter appears to conspire to form an isothermal profile in its inner regions, similar to that observed for spiral galaxies. Finally, upper limits have been set on the inner dark-matter profile of E/S0 as well as the (an)isotropy of the stellar component, but we defer a discussion until the entire sample has been analyzed.

\section{The value of $\mathbf{H}_{0}$ from lens time-delays}

The most severe degeneracy in lens models is that of the (unknown) slope of the radial mass profile of the lens mass distribution. Different power-law slopes (other than e.g. isothermal) can often equally well fit the same lensing-only constraints (e.g. Wucknitz 2002). Different slopes, however, lead to different 
inferred values of $\mathrm{H}_{0}$ from time delays, roughly following $\Delta \mathrm{H}_{0} / \mathrm{H}_{0}^{\gamma^{\prime}=2}=\left(\gamma^{\prime}-\right.$ 2 ); i.e. steeper (shallower) than isothermal mass profile lead to higher (lower) inferred values of $\mathrm{H}_{0}$.

Since the combination of stellar kinematics and gravitational lensing can tightly constrain $\gamma^{\prime}$ (see above; assuming the mass profile indeed follows approximately a power-law), the usefulness of this to time-delay lenses and the determination of $\mathrm{H}_{0}$ is apparent. We have thus far looked at two systems in detail and find $\mathrm{H}_{0}=59_{-7}^{+12} \pm 3 \mathrm{~km} \mathrm{~s}^{-1} \mathrm{Mpc}^{-1}$ (PG1115+080) and $\mathrm{H}_{0}=$ $75_{-6}^{+7} \pm 4 \mathrm{~km} \mathrm{~s}^{-1} \mathrm{Mpc}^{-1}(\mathrm{~B} 1608+656)$ for $\left(\Omega_{\mathrm{m}}, \Omega_{\Lambda}\right)=(0.3,0.7)$. The errors are the $68 \%$ C.L. and systematic errors and include a realistic uncertainty due to the slope of the radial mass profile. These values should therefore be relatively unbiased. The deviation of PG1115+080 from isothermal (see above) increases $\mathrm{H}_{0}$ by $\sim 35 \%$ from 44 to $59 \mathrm{~km} \mathrm{~s}^{-1} \mathrm{Mpc}^{-1}$, exemplifying the need to measure the stellar kinematics of lens galaxies for each time-delay system. Both values are consistent with local determinations of $\mathrm{H}_{0}$ (e.g. Freedman et al. 2001), but inconsistent with the sample studied by e.g. Kochanek \& Schechter (2003; and references therein). We are currently observing more systems with Keck and the VLT to improve the statistics of the sample and further examine the nature of this apparent inconsistency between lens systems.

\section{Conclusions}

The combination of stellar kinematic with gravitational lensing provides a powerful new tool to study the internal structure and evolution of E/S0 galaxies. Some of the preliminary results from the LSD Survey indicate that E/S0 lens galaxies to $z \sim 1$ evolve passively and have nearly-isothermal luminous plus dark matter mass profiles inside several $R_{\text {eff. Application of this to lens }}$ systems with time-delays gives more accurate values of $\mathrm{H}_{0}$, sofar in agreement with local determinations. The study of more lens systems is required to confirm/strengthen these conclusion, but results so far have been encouraging.

\section{References}

Freedman, W. L. et al. 2001, ApJ 553, 47

Kochanek, C. K. \& Schechter, P. L. [astro-ph/0306040].

Koopmans, L. V. E. \& Treu, T. 2002, ApJ 568, L5

Koopmans, L. V. E. \& Treu, T. 2003, ApJ 583, 606

Koopmans, L. V. E., et. al. 2003, ApJ submitted, [astro-ph/0306216]

Treu, T. \& Koopmans, L. V. E. 2002a, ApJ 575, 87

Treu, T. \& Koopmans, L. V. E. 2002b, MNRAS 337, L6

Wucknitz, O. 2002, MNRAS 332, 951 